\title{
Vibrotactile pattern recognition and discrimination at several body sites
}

\author{
ROGER W. CHOLEWIAK \\ Princeton University, Princeton, New Jersey \\ and \\ JAMES C. CRAIG \\ Indiana University, Bloomington, Indiana
}

\begin{abstract}
Although many body sites have been tested for the processing of vibrotactile patterns, very few studies have employed similar patterns and techniques to compare processing across loci. In the studies reported here, subjects judged comparable sets of spatial patterns presented in the same way to the finger, palm, and thigh in tests of recognition, discrimination, and masking. Discrimination varied as a function of stimulus onset asynchrony in a similar way across loci, but level of performance across sites depended, to different degrees, on duration. These differences were mirrored in the relative levels of recognition learning across sites. Levels of forward and backward masking on the fingers and thigh were less dependent on duration and site.
\end{abstract}

In attempts to develop prosthetic devices for the deaf and the blind using the skin as the alternative sensing system, many body sites have been examined. Most of the early systems, such as braille used by the blind and Gault's (1927) teletactor for the deaf, presented patterns to the fingers. In the past 20 years, with the development of dynamic arrays for tactile pattern presentation (Craig \& Sherrick, 1982), other sites have been examined, in part to allow free movement of the hands for other functions and in part so that larger tactile arrays could be used. Engelmann and Rosov's display (1975) and Brook's (Brooks \& Frost, 1983) multipoint tactile aid were designed to present analogues of the acoustic waveform in a unidimensional array to the volar forearm. Saunder's (1974) linear electrocutaneous belt and Sparks, Kuhl, Edmonds, and Gray's (1978) two-dimensional multipoint electrotactile speech aid (MESA) displayed similar electrocutaneous patterns to the abdomen. The tactile vision-substitution system (TVSS) (Bach-yRita, 1970) was designed to present optical patterns to the back. Similarly, the optical to tactile converter (Optacon) (Bliss, 1970) presented patterns obtained from text registered by a camera to the fingertip. In this listing of the variety of sites tested, the choice of location often was constrained by the physical

This research was supported by NIH Grant NS-04755 and NIH Grant NS-09783. Portions of this paper were read at a special symposium honoring Frank $A$. Geldard at the meetings of the Eastern Psychological Association in Baltimore, MD, April 14, 1984. The authors would like to express their appreciation for the careful reading and helpful comments of $\mathrm{C}$. E. Sherrick. Requests for reprints should be sent to Roger W. Cholewiak, $\mathrm{Cu}-$ taneous Communication Laboratory, C332 Engineering Quadrangle, Princeton University, Princeton, NJ 08544. dimensions of the display, the size of which was often determined, in turn, by the size of the transducers available (see, e.g., Scadden, 1973, p. 333). With developments in piezoelectric materials and miniaturization in the areas of both electronic circuitry and packaging technology, such limitations may be avoided. The choice of skin site, then, can be made on such other bases as its absolute sensitivity, its processing characteristics, or its cosmetic appeal, an important factor for the handicapped. One of the major reasons for the choice of the fingertip, for example, as the site of stimulation for the Optacon was its very high vibrotactile sensitivity and spatial acuity relative to other body sites. There have been, however, few attempts to examine pattern processing at several body sites to test those variables important to the rapid and accurate presentation of tactile patterns. Lacking evidence to the contrary, investigators have often had to assume that results obtained at one site of stimulation would be obtained at other sites.

There are, of course, many differences associated with changing the site of stimulation, such as density of innervation, relative number and type of neural units, and size of the homogeneous area of skin. Also varying are the uses to which the area is ordinarily put, such as grasping or palpating objects by the hand and fingers versus registering pressure by the thigh or back. These differences and others suggest (1) that the assumption of similarity of processing at different sites may be unwarranted, and (2) that it will be difficult to attribute any differences found among sites to a single cause.

Of the published studies that have tested several loci on the body with comparable tactile stimuli and procedures, the majority have been concerned either 
with threshold measurements of various kinds or with determining the growth of sensation magnitude or perceived distance with changes in one of the physical dimensions of the stimulus. In the first category fall the studies of Weber $(1826 / 1978)$, in which several types of spatial acuity for punctate pressure stimuli were tested over dozens of sites, and the studies of Weinstein (1968), in which comparable measures (including those for two points and for orientation) were made with improved procedures. Similarly, Verrillo (1966) has examined vibrotactile sensitivity in both glabrous (hand) and hairy (forearm) skin. He found that thresholds on hairy skin were 10 to $20 \mathrm{~dB}$ greater than those on glabrous skin in the frequency range of 25 to $450 \mathrm{~Hz}$.

In the second category are Verrillo's (1974) studies of the growth of magnitude of vibratory stimuli presented to the fingertip, palm, forearm, and thigh. In that series, both the absolute threshold and the slope of the functions varied with site in a complex fashion, interpreted as reflecting the density of underlying "neural units." Likewise, Green (1982) examined the perception of tactile distance for pressure stimuli presented to several body loci, showing that anisotropy in perceived distance was clearly demonstrable on the forearm but not on the thigh, palm, or abdomen, and that all sites showed a compression of perceived distance.

As far as we know, the only published studies comparing performance on patterned stimuli for several body sites are those of Engelmann and Rosov (1975), who used a unidimensional vibrotactile array to present tactile analogues of the speech signal, and Craig (1977), Scadden (1973), and Loomis (1980), who presented two-dimensional displays, recorded with camera systems, to the skin through vibrotactile matrices. Engelmann and Rosov (1975) presented linear spectral analyses of speech to the arms or thighs of observers who attempted to repeat the spoken stimulus. Transfer appeared to be extensive between the two sites tested: comparable levels of performance were achieved within a few test trials after the shift. In his paper describing the extraordinary ability of two observers to identify vibrotactile patterns using the Optacon, Craig (1977) noted that their performance on the abdomen, using a $10 \times 10$ element tactile array, was as remarkable as that on the finger. Similarly, "ordinary" observers' lower level of performance on the finger was reflected in lower performance on the abdomen. Scadden's study (1973) used a single device (in this case, the TVSS), the same patterns, and the same observers to examine the transfer of performance across body sites. Observers trained to perceive patterns presented to the back were quickly able to learn to identify patterns presented to the abdomen or inner thigh. In fact, within the $\mathbf{4 0}$ test trials, their performance using the other sites was superior to that with the back. Loomis (1980) also reports comparable letter-recognition scores for patterns presented with the TVSS to the back, abdomen, and thigh.

Like these previous studies, the present study was concerned with examining changes in levels of performance with changes in body site, although we realized that it might be difficult to attribute these changes to any single factor. We were also concerned with the processes underlying pattern perception and functional changes that might accompany changes in location. For the functional changes, we were less concerned with the levels of performance than with the change in performance as we manipulated the same variable at each of the sites examined. Specifically, we tested the effect of time between successive patterns both in a discrimination task (Experiment 1) and in a masking paradigm (Experiment 3 ). Recognition performance, which might underlie the discrimination functions, was also measured (Experiment 2). Such a series of studies has a direct bearing on the relative suitability of sites for a communication system, because such a system would necessarily present a sequence of vibrotactile patterns for the purpose of conveying an extended message in real time.

The experiments examined these types of timedependent processing for vibrotactile spatial patterns presented to several body sites: the thigh, palm, and fingertips. Measurements on the thigh and palm were conducted at Princeton University by the first author. Measurements on the fingertips were performed at Indiana University by the second author, who had already begun to examine the role of stimulus onset asynchrony (SOA) in the discrimination of tactile patterns (see Craig, 1983).

\section{GENERAL METHOD}

The system used to generate the patterns presented to the palm and thigh consisted of a square array of 64 piezoceramic benders controlled by a Digital Equipment Corporation PDP-8/f computer. Different arrays were used for the two sites tested. The thigh matrix was made up of 5-mm-diam contactors on 15 -mm centers in a square $8 \times 8$ array. The matrix could be adjusted to fit the contour of the left thigh of the seated observer, resting with a static force of approximately $10 \mathrm{~g}$ per contactor. The smaller, palm matrix was made up of $2-\mathrm{mm}$ contactors on approximately 8-mm centers. The heights of the contactors were adjusted to approximate the curve of an "average" left palm, but could not be adjusted for each observer. The hand rested on the array, palm down, with a small sandbag placed over it for stability. The patterns of vibration on the matrices were specified by computer commands controlling the locus, intensity, and duration for the active elements, vibrating at $230 \mathrm{~Hz}$. A more complete description of the apparatus may be found in Cholewiak and Sherrick (1981).

The Indiana system was used for pattern generation and data collection when the fingertip was the stimulus site. It consisted of a PDP-11/34 computer interfaced with a 144-element tactile array (adapted from the Optacon), which measures $1.1 \times 2.7 \mathrm{~cm}$ and fits against the distal portion of the observer's fingerpad. The elements of the array are arranged in a matrix, 6 columns $\times 24$ rows. Only the upper (distal) 18 rows were used for the patterns. When energized, the elements vibrate at $230 \mathrm{~Hz}$, the same frequency used on the palm and thigh matrices. Additional details of this apparatus may be found in Craig (1980).

To facilitate comparison among body sites, the stimulus pat- 


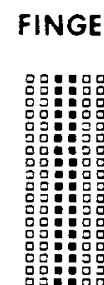

$$
\begin{aligned}
& \text { EQUAL } \\
& \text { NUMBERS }
\end{aligned}
$$
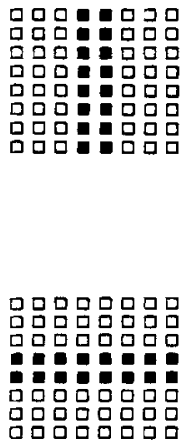

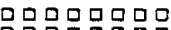

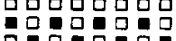

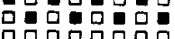

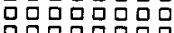

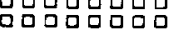
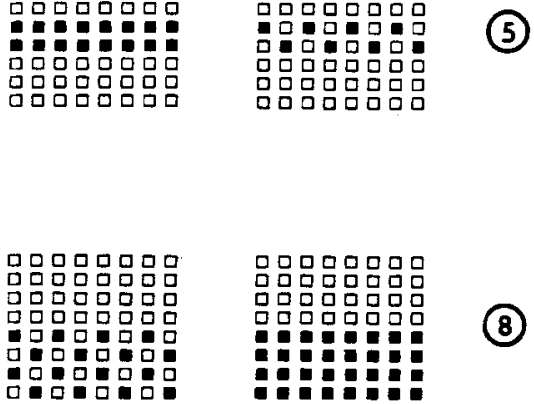

(8)

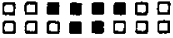

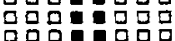

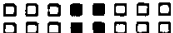

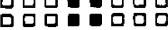

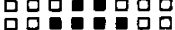

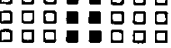

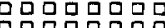
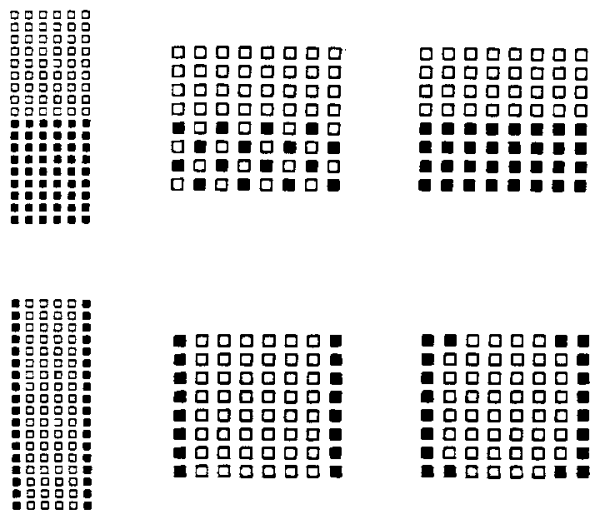

Figure 1. Representative patterns from the sets used in the studies reported here. In column 1 are represented the patterns used In the studles on the fingertips using the Optacon array. The second and third columms show the comparable patterns designed for the palm and thigh displays with equal or unequal numbers of active elements.

terns and procedures in the thigh and palm experiments were made as similar as possible to those in the fingertip studies. The patterns used in these studies were a set of " 10 easy patterns," so named because identification rates typically exceeded $90 \%$ within 100 presentations to the index fingerpad with the Optacon system (see, e.g., Craig, 1983). The patterns for the thigh and palm were modified somewhat to fit the $8 \times 8$ element vibrotactile matrices. Representative patterns from both sets are illustrated in the first two columns of Figure 1.

\section{EXPERIMENT 1: PATTERN DISCRIMINATION}

Experiment 1 measured discrimination of spatial patterns presented to the palm and thigh as a function of the time between the patterns. The measurements were made to determine how discriminability changes as a function of the time between patterns, to see how the overall level of discriminability is affected by a change in the site of stimulation, and to compare these results with similar measurements made on the fingertip by Craig (1983).

Determining how well pairs of patterns can be dis- criminated and how their discriminability is affected by the time between compared patterns provides basic information about the rate of information transmission and the processing of sequences of patterns. For example, Craig (1983) found that with reduction of the time between patterns presented to the fingertips, there was a decline in discrimination performance. This suggests a limit on the rate at which successive patterns could be processed. Using pairs of patterns that differed in duration, Craig also found that the data could be fit by a single line when plotted as a function of stimulus onset asynchrony (SOA) rather than some other measure of temporal separation, such as interstimulus interval (ISI). The implication is that if patterns are equated for perceived intensity, as they were in the Craig study, then neither pattern duration per se nor amount of temporal overlap affects discriminability. The question of whether such a relationship holds across body sites was examined here.

\section{Method}

Four naive observers served in this discrimination task, which involved the use of pairs of patterns drawn from the 10 already described. Representative patterns are shown in the middle column of Figure 1. The 90 possible pairs and orders were intermixed with 90 pairs in which the first pattern was the same as the second ( 9 repetitions of each of the 10 patterns). For each session, the pattern duration was fixed at either 4 or $52 \mathrm{msec}$, and one-quarter of the possible 180 pairs were selected for testing. The pattern pairs were presented at each of six SOAs: approximately $10,17,26,56$, 96 , and $300 \mathrm{msec}$. The exact times depended on the pattern duration and are indicated on the resulting discrimination functions. Over the four sessions, each observer made 180 judgments at each of the six SOAs. In two of the sessions, the palm was the site of stimulation; in the other two sessions, patterns were presented to the thigh.

The observer responded "same" or "different" by pressing one of two buttons on a keypad connected to the PDP-8/f computer, which automatically recorded the responses. In the experimenter's instructions, accuracy was emphasized over speed. Feedback was given after correct responses in the form of a tone over the white noise in the observer's headphones. In addition, after each response, a visual display presented a " 1 " or a " 2 " for "same" or "different" stimuli, respectively, followed by a " 1 " or " 2 " to indicate the observer's response. The two numbers matched when the response was correct. Sessions involving the palm were preceded by a presentation of sequential taps at each of the 64 contactors in the array to insure adequate contact over its surface, a procedure that was not necessary with the larger, adjustable thigh matrix. For both the palm and thigh, the patterns that were $52 \mathrm{msec}$ in duration were equated in perceived intensity with the 4-msec patterns by reducing the intensity of the longer patterns to a level determined by a brief preliminary matching series. At either of these sites, patterns were matched in loudness by equating the numbers of active elements in the set. Cholewiak (1979) has shown that number of active elements in such an array, rather than pattern density or proximity, determines overall pattern loudness when each element is presented at the same intensity.

\section{Results and Discussion}

The data were collapsed into a $2 \times 2$ stimulusresponse matrix across patterns for each SOA, and a total corrected percent correct, $P(C)_{\max }$, was calculated with a computer algorithm for each SOA. This 


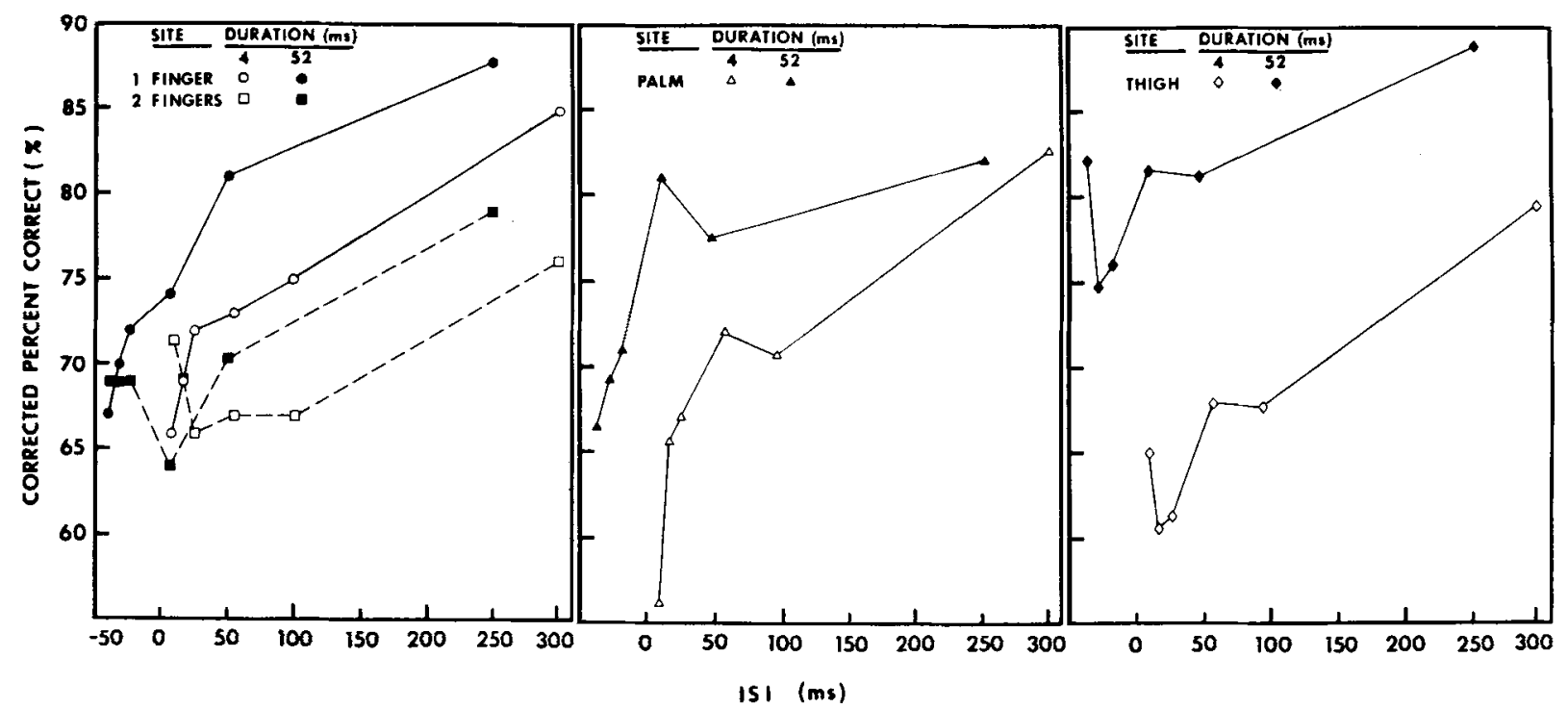

Figure 2. Discrimination performance for fingers, palm, and thigh as a function of interstimulus interval (ISI). Two pattern durations were tested, 4 and $52 \mathrm{msec}$, as shown for each site. Finger data are reproduced here from Craig (1983).

statistic minimizes effects of response bias (McFadden, 1970) and was obtained with a procedure that takes into account the observer's hit and false-alarm rates from the response matrix. Specifically, after these rates were obtained for an SOA, the " $Z$-score" was calculated for each, $d$ ' (an index of discriminability) was derived from them, and $P(C)_{\max }$ was recovered by converting the resulting $d^{\prime}$ back to a percentage. These data were then plotted as a function of both ISI and SOA in Figures 2 and 3, respectively, across body sites and durations.

For comparison, the data from Craig (1983) for a single finger (filled and open circles) and for two fingers (filled and open squares) have also been plotted. Although the original Craig paper determined $\mathbf{P}(\mathbf{C})_{\max }$ by reference to tables of $d^{\prime}$ (see Swets, 1964), the points plotted here show $P(C)_{\max }$ calculated as above. The values obtained by the two methods differ by less than $2 \%$, on the average. Note that SOA and ISI are plotted on linear scales to facilitate comparison, although SOA is typically plotted on logarithmic coordinates.

The results suggest several conclusions. First, the data from all three sites indicate a general improvement in discriminability with increasing temporal separation which has, for the finger, been attributed to a reduction in masking, a point that will be considered in Experiment 3. Second, the overall shapes of the functions for the two different pattern durations are notably similar when plotted as a function

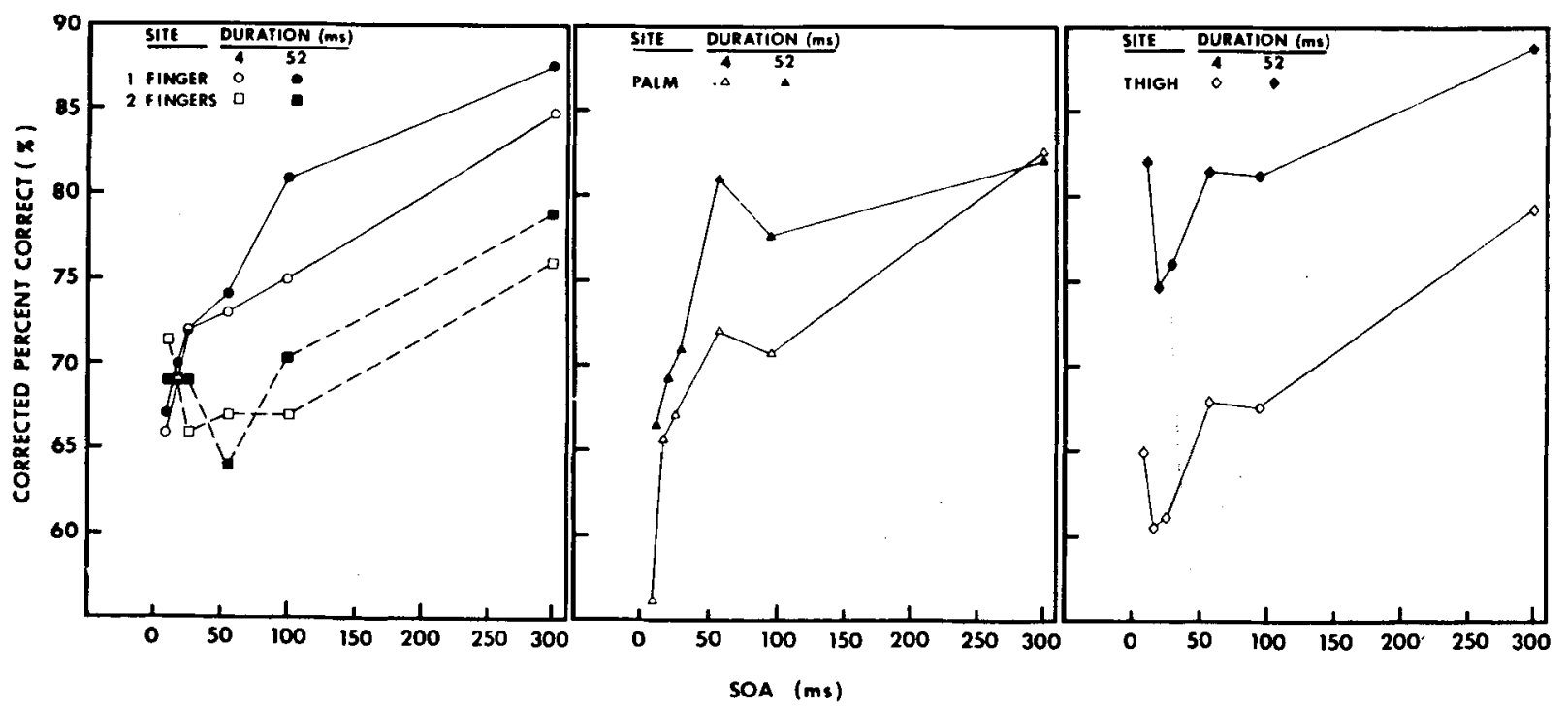

Figure 3. Discrimination results from Figure 2 replotted as a function of stimulus onset asynchrony (SOA). 
of SOA. For example, both the 4- and 52-msec SOA functions on the thigh show a minimum at $9 \mathrm{msec}$, with performance improving as the SOA decreases to $4 \mathrm{msec}$. Plotting these data as a function of ISI shows the minima and upturn now occurring at different temporal separations, depending upon pattern duration. The general similarity of the SOA functions suggests that pattern onset may enjoy some functional significance not shared by ISI in determining accuracy of discrimination.

Third, there is an effect of pattern duration on the absolute level of performance. Performance is better for the 52-msec pattern pairs than it is for the 4-msec pairs, particularly when the patterns are delivered to the thigh and palm. The mean difference between the 52- and 4-msec patterns increases, as one goes from finger to palm to thigh, in the amounts of $1.8 \%$, $5.7 \%$, and $13.7 \%$, respectively. For the thigh and palm, particularly, performance levels are influenced by both pattern duration and SOA: Duration affects overall level, whereas SOA determines the shape of the functions better than ISI. Some potential sources for the difference in performance between the two durations, such as perceived intensity, were equated and thus controlled for in this experiment.

The differences in the absolute level of discrimination performance across sites may be the result of several factors already mentioned, such as pattern size and density, perceived pattern intensity, density of innervation at a site, and so on. To show the degree of parallelism in the functions obtained, across sites, the results for the 4-msec patterns were equated at $300 \mathrm{msec}$ and the functions replotted in Figure 4a. The 52-msec data were replotted in the same fashion in Figure 4b. The 4-msec functions show a fair degree of similarity across sites when compared in this manner, certainly more than the 52-msec data. Yet the slopes of the functions, particularly for the longer SOAs, are quite similar. In summary, the improvement in performance as a function of SOA is comparable across sites; however, some site-related differences remain even after absolute levels of performance are equated.

The improvement in performance at the briefest temporal separations for patterns presented to the thigh was seen with three of the four observers; the fourth observer showed a decline in performance at the shortest SOAs. This improvement at briefer times has been seen in a discrimination task before when the patterns were presented to two fingers (see Figures 2 and 3 ). In the case of two fingers, the improvement was less pronounced and occurred over somewhat longer temporal intervals. It may be that, at these briefer intervals, additional cues such as apparent motion (Sherrick \& Rogers, 1966) or cutaneous saltation (Geldard, 1975, 1982; Geldard \&

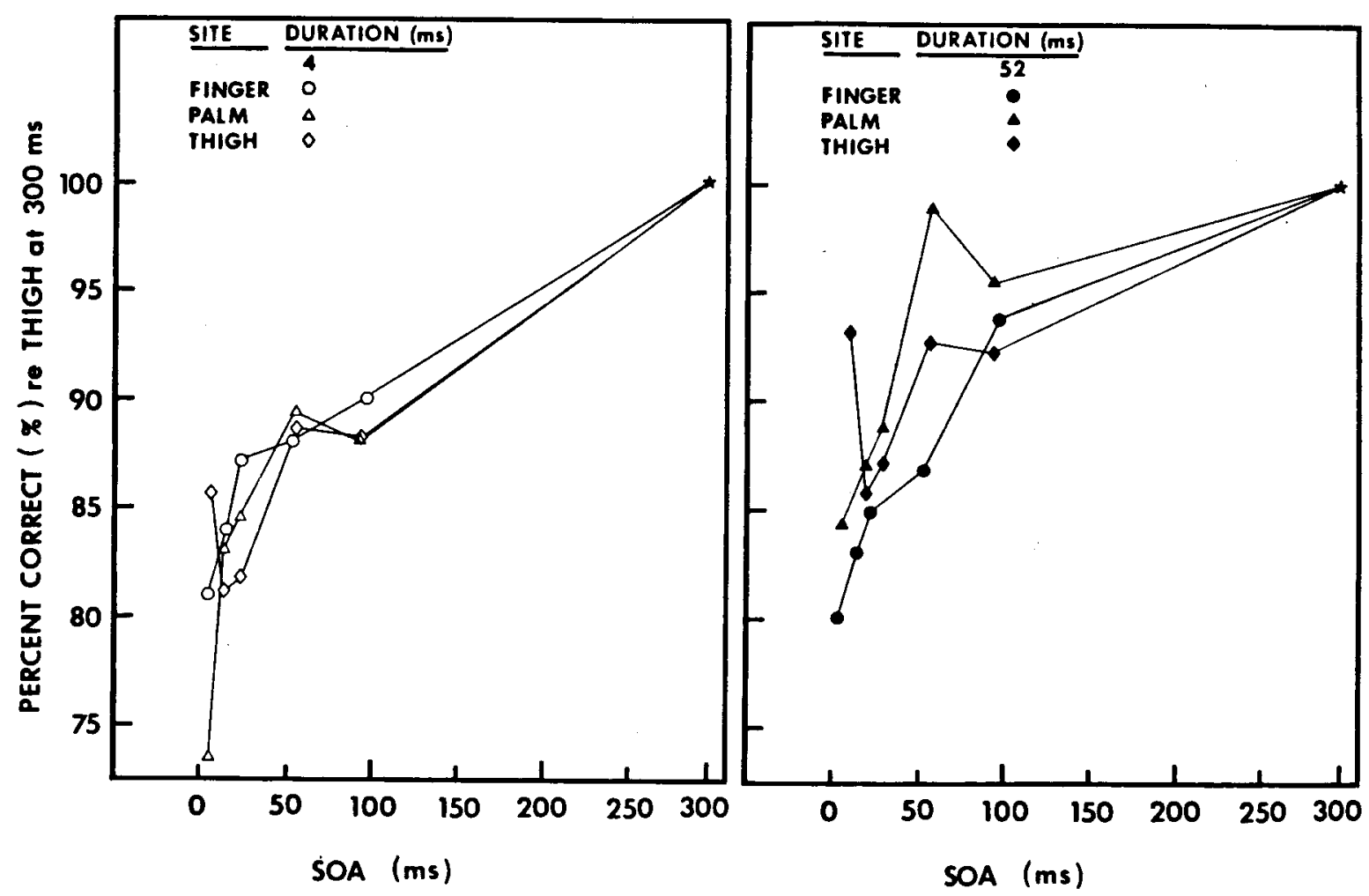

Figure 4. Discrimination performance for finger, palm, and thigh plotted as a function of stimulus onset asynchrony (SOA), normalized to performance at $300 \mathrm{msec}$. Data are shown for both 4 and $52 \mathrm{msec}$ durations. Finger data are from Craig (1983). 
Sherrick, 1972) may be producing the improvement in performance on the thigh and on the two fingers. These cues may not be as salient when patterns are confined to a smaller area, such as the palm or a single finger.

In one attempt to explain discrimination, Craig (1983) has proposed that such performance reflects the joint probability of correctly identifying both patterns (remembering that the two patterns may mask one another) plus the probability of chance performance (contributing $50 \%$ to the total correct response rate). Such calculations, however, have generally underestimated performance at all but the longest SOAs. The presumption is that, besides the ability to identify correctly each member of the pair, some additional characteristics of the display provide cues to improve performance. Features such as movement, discussed above, or intensitive changes (possibly owing to temporal integration or "fusion" of the two patterns at very short SOAs) could provide just those cues. Indeed, many observers volunteered that they used such criteria to some advantage. One consistent feature in the observers' reports was the tendency to use different strategies for different stimulus conditions. At shorter SOAs, for example, certain "different" patterns would produce a strong sensation of movement across the array or an increase in perceived intensity. Other phenomena emerged with other pairs of stimuli, some of which, like movement, were obvious and useful only at shorter intervals, whereas others provided cues at longer SOAs. Without these additional cues, presumably performance would be even worse at the shorter SOAs.

The change in the available cues might lead observers to modify their criteria for "same" versus "different" as SOA varied. Indeed, the greater tendency to respond "same" at shorter SOAs (indicated by same/different response ratios averaging 1.24 for SOAs of 29 msec or less) suggests that factors such as temporal integration may be biasing responses. For longer SOAs, on the other hand, the average of same/different response ratios is 0.97 , reflecting little if any response bias.

Pattern duration has a large effect on discrimination performance for the thigh, less so for the palm, and even less so for the finger. This fact indicates that equating different duration patterns for equal perceived intensity is effective in producing equal discrimination performance only on the finger. Given the similarity in slopes for the 4- and 52-msec functions when plotted as a function of SOA, it would be expected that if some means could be found for equating performance across duration, then a single function might be fit to the data. Regardless of site, however, the time between onsets of successive patterns is the critical variable in determining changes in discrimination performance with temporal sepa- ration of patterns; the level of performance, on the other hand, apparently is affected by factors related to the site of stimulation.

It is difficult to make specific statements regarding the absolute levels of performance across sites (because of unavoidable differences that existed along several dimensions, such as pattern size and stimulator density). However, the relative differences in discrimination, particularly at shorter pattern durations, might be a reflection of the decrease in sensitivity and acuity associated with the change in locus from fingertip to palm to thigh. Such siterelated differences should be reproduced in recognition performance. The results of a masking task might also help to explain the differences in discrimination because of the similarity in dependence on temporal factors shared by the two. These issues will be examined in greater detail in Experiments 2 and 3.

\section{EXPERIMENT 2: PATTERN RECOGNITION}

What could contribute to the differences both within and across sites seen in Experiment 1? As we have suggested, discrimination performance might be based on the ability to recognize the individual patterns and then to discriminate between them by a process of comparison. One might suspect that if discrimination depended upon successive identifications of individual stimuli, superior recognition should lead to superior discrimination, Experiment 2 examined whether the differences in discrimination are reflected in, and possibly based on, differences in recognition performance.

\section{Method}

In this series, sets of stimuli that consisted of each of the 10 patterns described above (shown in Figure 1, column 2) were created. These were presented individually for either 4 or $52 \mathrm{msec}$, the two durations used in the discrimination experiment. Each pattern was represented 10 times in a randomized series of 100 presentations. Four such blocks of trials were presented to each naive observer within a single session. Four groups of five observers were tested; the patterns were presented to either the palm or the thigh at pattern durations of either 4 or $52 \mathrm{msec}$. Pattern intensity was equated across duration by using the mean of the matches from Experiment 1 and setting the 52 -msec patterns to that level. Two additional groups of observers were tested, but with a 26-msec pattern duration, identical to that used by Craig (1983), to establish recognition baseline levels on the fingertips. One group was tested with patterns on the thigh, the other on the palm. Because pattern identification has been shown to be directly related to pattern intensity (Craig, 1980), the voltage to the stimulators was set to its highest value in an attempt to maximize performance for the 26msec series.

The observer responded by pushing one of 10 buttons on a telephone-type keypad marked with drawings of the patterns. The complete set of illustrations, like those in the middle column of Figure 1, was shown to the observer at the beginning of the session, and their similarity to those on the keys was noted. Feedback was provided after each response in the form of a visual display showing the number of the stimulus and that of the observer's response. If the response was correct, not only did the displayed 
numbers match, but also a tone was presented in the observer's headphones over the masking white noise. A short break was given between each block of trials. To control for response bias, the data were converted to $\mathrm{P}(\mathrm{C})_{\max }$, as before.

\section{Results and Discussion}

The results are shown as learning curves in Figure 5 for pattern durations of 4 and $52 \mathrm{msec}$. The data are shown for the two body sites, with means and standard errors indicated for each 100-trial block.

The hypothesis that the superior discrimination performance seen in Figure 3 for the 52-msec thigh condition is owing to superior identification for those patterns is not borne out. Indeed, based on the identification data shown in Figure 5, the ordering of the functions in Figures 2 and 3 would be predicted to be $\mathrm{P}-52, \mathrm{P}-4, \mathrm{~T}-52$, with $\mathrm{T}-4$ poorest, assuming that those observers were trained to recognize the individual patterns. It is notable, however, that the differences in discrimination performance at a particular site owing to pattern duration are predicted by recognition performance. At the end of the series of recognition trials, the means of the 4- and 52-msec-duration series differed by approximately $7 \%$ for the palm and $15 \%$ for the thigh (Figure 5). Comparable differences
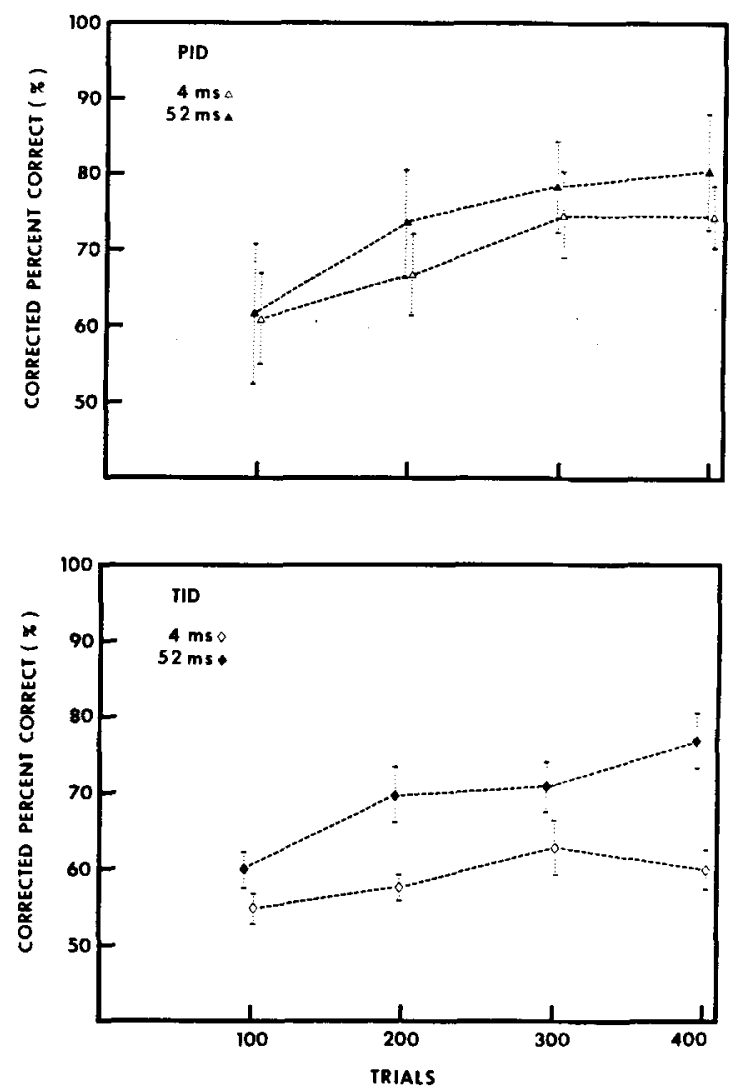

Figure 5. Recognition performance for the palm (PID) and the thigh (TID) plotted over blocks of 100 trials each. Means and standard errors of the means are given for pattern durations of 4 and 52 msec. are seen in discrimination performance plotted in Figure 3 over SOAs. Craig (1983) found little difference in identification performance for the 4- and 52msec patterns on the fingertip (when equated for perceived intensity), and, as Figure 3 shows, little difference is seen in discrimination performance. Thus, it appears that pattern duration affects both discrimination and identification in a similar manner at a given site. Nevertheless, predicting discrimination performance across sites based on identification scores appears to be difficult.

It was mentioned earlier that equating the perceived intensity of patterns presented to the finger for different brief durations results in approximately equal levels of recognition performance (Craig, 1980). For patterns presented to the palm, however, the data suggest that better performance is achieved for the longer patterns. This trend is more clearly evident for the thigh, even though, in both cases, the patterns have been equated for equal perceived intensity. This finding might be related to the size of the patterns across the three areas of stimulation and the relative ease with which subjects can integrate, both spatially and temporally, across these sites. It has been suggested that subjects may be able to process patterns presented to the fingertip in parallel, whereas patterns presented to such body sites as the back or abdomen are processed serially (Craig, 1980). If that is the case, then these latter sites, perhaps including the thigh, should presumably benefit from having patterns presented for a longer period of time.

One of the most surprising results (also supporting the last point) is the good discriminability for the 52msec patterns on the thigh. The upturn at the briefest SOA has already been noted; however, in light of the recognition results, it is also surprising that discrimination performance is as good as it is at the longest SOA, 300 msec. At such a temporal separation, few of the phenomena that might improve discriminability at briefer times are operating. One might well expect, then, that discriminability would be much more dependent upon identification at the longer SOAs. For the 4-msec patterns, the rank ordering of discriminability at the 300 -msec SOA follows the recognition results-finger, palm, thigh. In the case of the 52-msec patterns, however, performance is considerably better for the thigh than these identification results would predict.

As noted before, identification performance for the 10 patterns presented at durations of $26 \mathrm{msec}$ to the fingertips reached levels of $95 \%$ to $100 \%$ correct in the first 100 trials of testing (Craig, 1983). The comparable data on the thigh and palm with 26-msec pattern durations are shown in Figure 6. The data are plotted as they were in Figure 5. These functions clearly do not approach the levels achieved on the fingertip in the first 100 trials; however, the palm re- 


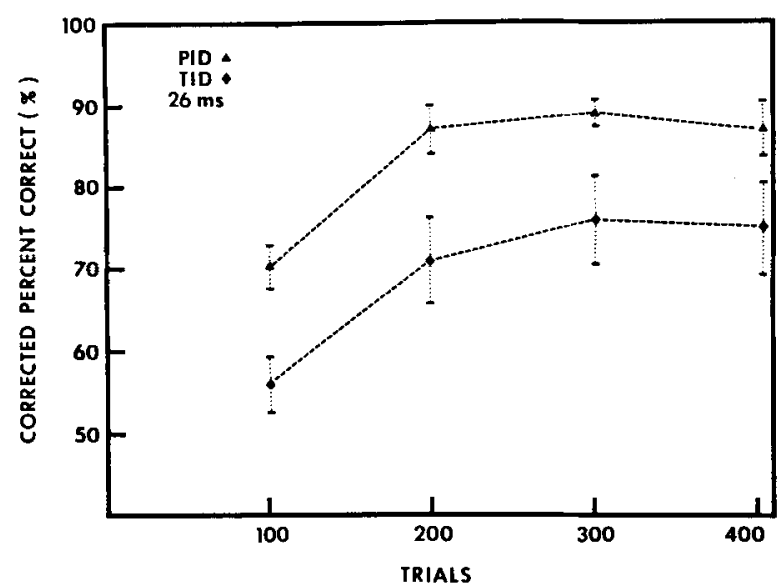

Figure 6. Recognition performance for the palm (PID) and the thigh (TID) plotted over blocks of 100 trials each. Means and standard errors of the means are shown for pattern durations of 26 meec.

sults do approach $90 \%$ correct by the second block of 100 trials.

In comparing the functions shown in Figure 6 with Craig's fingertip results, however, it needs to be noted that although the patterns presented to the thigh and palm consisted of the same numbers of active elements, the patterns presented to the fingertips were made up of numbers of active elements ranging from 12 to 54 . In these cases, the active contactors were presented at equal intensities. Varying the number of elements in such a pattern does produce changes in the perceived intensity of that pattern (Cholewiak, 1979), and thus for the patterns presented in that study to the fingertips, an additional redundant cue, perceived intensity, could have been present. It is possible that the lower level of performance seen in Figures 5 and 6 for the palm and thigh relative to that described for the fingertip might be owing to the absence of these cues rather than to any site-related characteristics. Two manipulations were performed to see whether such cues might be leading to better performance on the fingertips relative to the other sites tested: First, subjects identified the $\mathbf{1 0}$ patterns presented to the fingertips after these patterns had been equated for perceived intensity by adjusting the voltage applied to the tactile stimulation; and second, subjects identified patterns presented to the thigh when the number of elements comprising the patterns was varied to introduce an intensitive cue. The additional series of recognition trials on the thigh was run with a new pattern set, shown in Figure 1 (labeled "Unequal Number"). In this series, the numbers of elements per pattern are in the same ratios to one another as those presented to the fingertip (leftmost column).

The two additional groups of observers who judged these patterns at the 26-msec duration were tested in the same way as those in the last study. Five naive observers identified patterns presented to the thigh, and three observers were tested on the fingertip patterns. These three had participated in several tactile pattern perception tasks; however, none of them had had any prior experience with the particular patterns used. In both cases, recognition trials were completed within a single testing session. As before, feedback was provided on a trial-by-trial basis. The thigh sessions consisted of four blocks of 100 trials each, whereas the fingertip sessions consisted of six blocks of 50 trials each.

Three functions are shown in Figure 7. The first, indicated by open diamonds, represents the results for the thigh with the intensity covarying with pattern. The second, indicated by filled circles, represents the finger patterns of equal intensity. The third set of data (filled diamonds) are the equal-intensity thigh data from Figure 6 shown for purposes of comparison. The facts (1) that the two thigh functions are very similar, even though intensity could be a cue in one of the conditions, and (2) that performance on the fingertip patterns is still much better than at either the thigh or palm, even though intensity differences no longer exist, strongly suggest that intensitive manipulations had little effect in determining the relative levels of performance among thigh, paim, and fingertip. Recognition performance on the thigh is poorer than on either the palm or finger, and this lowered performance is apparently not owing to quantitative characteristics of the stimuli, such as pattern intensity. Experiment 2 still does not account for the fact that discrimination performance on the thigh can be superior to that on the other two sites, even though recognition performance is poorer.

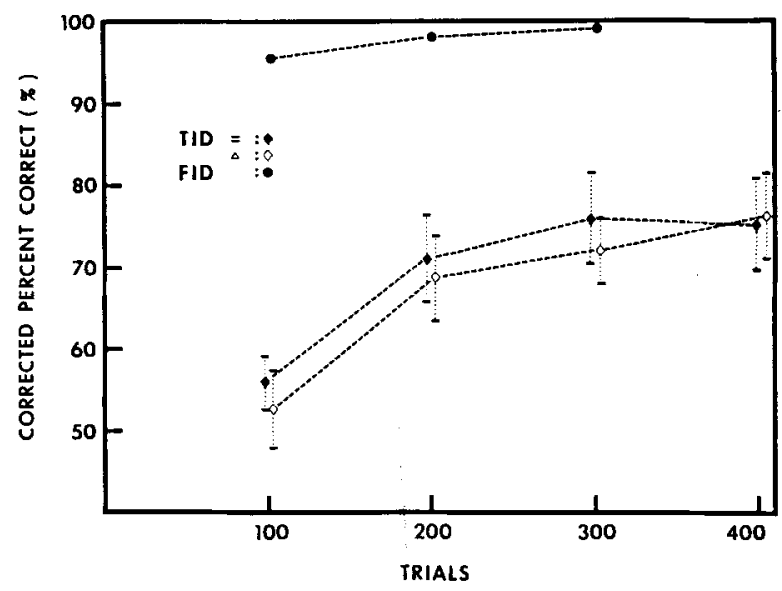

Figure 7. Recogaltion performance for the finger (FID) and the thigh (TID) plotted over blocks of 100 trials ench. Thigh function are shown for experiments in which the number of active elements across patterns were equal (=I) or different (AI). Means and standard errors of the means are plotted for pattern durations of 26 msec. 


\section{EXPERIMENT 3 PATTERN MASKING}

Although temporal masking has proven to be an important measure of processing capabilities, there are, as far as we know, no data on forward and backward pattern masking on any sites other than the fingertip, and no data comparing such masking functions across sites. Experiment 3 measured forward and backward masking for patterns presented to the thigh and compared these results to those obtained on the finger.

These temporal masking measurements were undertaken for several reasons. It is conceivable that the differences seen in discrimination in Experiment 1 may be the result of differences in the mutual interference (masking) of each member of the pair of patterns on one another. If the two patterns mask one another, discrimination performance would be expected to be poor. In fact, discrimination and masking performance might be more highly correlated with one another than either of these with recognition performance in the absence of a masker. This could be the case because in both discrimination and masking, patterns are judged in the presence of a temporally contiguous stimulus. On this basis, given that discrimination performance for the longer duration pair of stimuli on the thigh is very good (Figure 3), masking for these stimuli is predicted to be less than for comparable stimuli on the finger, where discrimination performance was somewhat poorer.

There is another reason to speculate that less masking might be seen on the thigh than on the fingertip. This has to do with the shape of the discrimination functions. As noted in Experiment 1, the improvement in discrimination at the briefest SOAs for patterns presented to the thigh was similar, although not identical, to results obtained when the patterns were presented to two fingers. Masking across the two fingers was considerably reduced as compared with masking on the same finger (Craig, 1983). Thus, on the finger, reduced masking is associated with a nonmonotonic, bow-shaped discrimination function. Does the same association hold for the thigh?

\section{Method}

A total of eight people participated in this study. Each had served as an observer in a recognition test for patterns of 26-msec duration (some of their data are represented in Figure 7). After $\mathbf{4 0 0}$ recognition trials, they began the masking series. This consisted of 100 trials/condition/session (400 trials/session) over two sessions, ending with another block of 100 recognition trials. By the end of the first recognition series $\mathbf{P}(C)_{\max }$ for this group of observers averaged $78 \%$ (standard error $=2.4 \%$ ). In the postmasking series, data for only six observers were available, but they averaged $79 \%$ (standard error $=2.5 \%$ ). . No changes in recognition capability occurred as a result of the experience on the masking trials.

In the masking experiment proper, the procedures were similar to those used in the discrimination study. Depending on the instructions from the experimenter at the start of a series, however, the observer was to identify either the first pattern in the pair (backward masking condition) or the second pattern in the pair (forward masking condition). Each pattern was presented for $26 \mathrm{msec}$, and the SOA between masker and target patterns was either 50 or $100 \mathrm{msec}$, resulting in either a 24 - or 64 -msec ISI. As in the preceding experiments, both auditory and visual feedback were provided after each response. Every pattern was combined with every other pattern (including itself) as masker and target, and the complete set was judged twice by each observer.

\section{Results and Discussion}

When the corrected percent correct responses were calculated for these sessions, and then subtracted from the baseline response rate for recognition $(78 \%)$, the function shown as a solid line in Figure 8 results. Means for the eight observers are shown along with standard errors of the means (1,600 observations per point). Also plotted are the masking functions from Craig's (1983) experiment when the "10 easy patterns" were presented to a single finger or to two fingers. In the first case, both target and masker were presented to the index finger. In the second case, the target was presented to the index finger and masker to the middle finger. The fingertip data from Craig (1983) were recalculated as $\mathrm{P}(\mathrm{C})_{\max }$ in the manner described above to allow for direct comparison with the thigh data. The differences between these values and those in the original paper were less than $2 \%$.

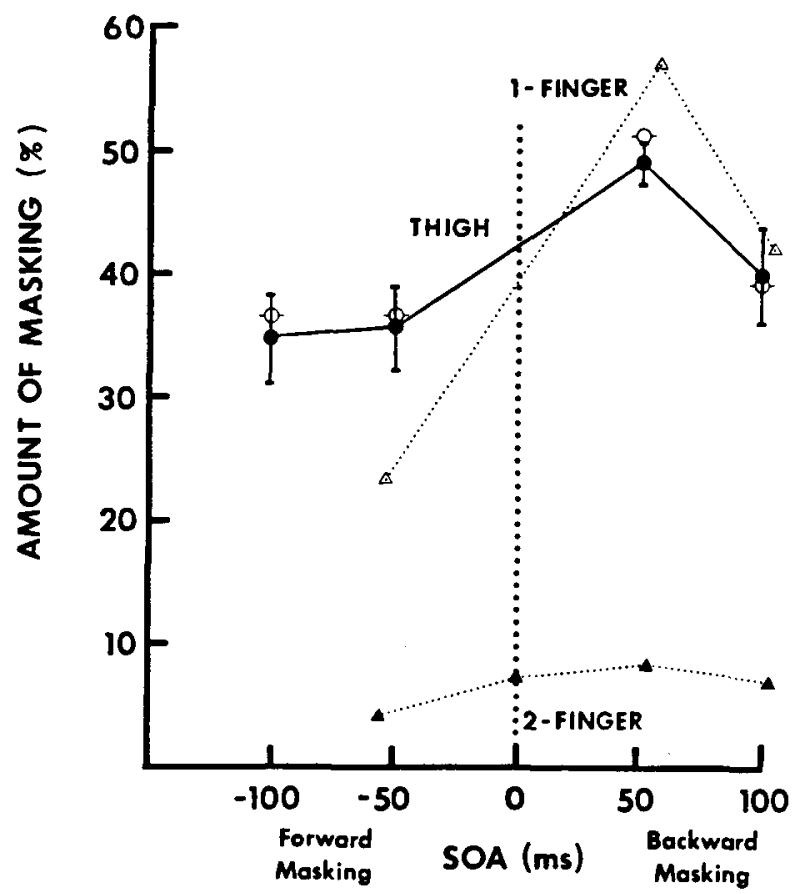

Figure 8. Amount of making as a function of the atimulus onset asynchrony (SOA) between the target and masker. Masking was measured as the reduction in $\mathbf{P}(C)_{\max }$ from a no-musker recognition condition. Pattern duration was 26 msec for the data indicated by solid symbols and 52 msec for the thigh open-eircle data. Finger data are from Craig (1983). 
Both the thigh and finger functions reveal more backward than forward masking, although the difference between the two types of masking is not as pronounced when patterns are presented to the thigh. Using estimates from Craig's (1983) data, one would expect that extrapolating the forward masking function for a single finger to $-100 \mathrm{msec}$ would result in an additional decline of approximately $7 \%$, increasing the difference between the thigh and the finger functions. The implication is that there may be a difference in the slopes for the forward masking functions from these sites. It is, however, clear that the thigh masking function is much more similar to the one-finger than to the two-finger function, yet the similarity of thigh to finger discrimination functions is just the reverse. One conclusion is that despite the similarities in the results from the first experiment for these sites, the process underlying discrimination and masking are different.

The patterns were presented for $26 \mathrm{msec}$ to be comparable to the conditions tested on the fingertips. It might be argued that had patterns of 52-msec duration been used, that is, the pattern duration that led to good discrimination performance in Experiment 1, there might be a dramatic decline in the amount of masking. An additional five observers were tested with 52-msec-duration target and masking patterns, and the results obtained for the same SOAs showed the mean amounts of masking to be identical to those for $26 \mathrm{msec}$. These data are also plotted in Figure 8, as open circles. Note that in these cases ISIs were either -2 or $48 \mathrm{msec}$ for the 50- and 100-msec SOAs. Actual overlap of the patterns occurred only in this 50 -msec condition, and only for the $2 \mathrm{msec}$ indicated.

The data from Figure 8 indicate that backward masking on the thigh is comparable (if not slightly lower in magnitude) to that on a single finger. The forward masking functions show much poorer performance (greater amounts of masking) for the thigh, ranging from more than $10 \%$ at $-50 \mathrm{msec}$ to over $20 \%$ (extrapolated) at $-100 \mathrm{msec}$, when compared with similar conditions on the finger. Yet the data from the first experiment demonstrated that, at least for 52-msec patterns, same-different discriminations are more accurately made on the thigh. One implication is that little, if any, forward masking enters into this type of judgment; otherwise, discrimination of patterns presented to the thigh would be poorer than for comparable patterns presented to the finger. Only backward masking may play a role, if masking is involved at all. These results are all the more surprising in that recognition is so much poorer on the thigh (and on the palm) than on the finger. That masking on the thigh (or on a single finger) is so much greater than that on two fingers is to be expected, because in the case of two fingers spatial interactions are eliminated. In addition, because the target was always presented to the same finger, ob- servers could focus attention only to that site, further reducing any potential interference.

\section{GENERAL DISCUSSION}

As noted before, the differences in levels of performance reported across sites for the three experiments may have several sources. One of these is that observers who were tested on the palm or thigh were completely naive to such tasks, whereas those tested on the finger were both prescreened and had had some experience judging vibrotactile patterns. This would tend to produce, at the least, a reduction in the variance and perhaps an overall improvement in performance. Furthermore, the notion of " 10 easy patterns" may be applied only to those individuals who have demonstrated abilities in recognizing vibrotactile patterns. On the other hand, the screening procedure used with the subjects tested on the fingertip patterns was not very selective (over two-thirds of those tested passed the screening procedure). Also, most of the change in performance seen in the present experiments occurred in the first several hundred trials (Figures 5, 6, and 7), suggesting that continued experience might well improve performance but not enough to change the overall rank ordering of the results across sites. In fact, the best performance recorded in the discrimination series (Experiment 1) was recorded with naive observers judging 52-msec thigh patterns. However, the predictive power of the several tests on one another might differ considerably, depending on the prior experience of the populations tested. It is even possible that the predictive power of one test on another might drop with a preselected group of observers, depending on the types of skills selected for and their usefulness in the test battery.

There are, in addition, some differences in the devices used to generate the patterns that may have interacted with the sets of patterns such that those tested on the finger were easier to learn than those on the palm or thigh. The original set of patterns was designed to take advantage of the vertical orientation of the Optacon display. A set of " 10 easy patterns" created specifically for the square arrays would probably look somewhat different and be less biased towards horizontal patterns. It is possible that any improvement in recognition resulting from the use of such a set would produce comparable improvements on the other measures, although, as we have seen, varying pattern intensity does not have this effect. Finally, the sites themselves differ on many dimensions, including skin type ("hairy" versus glabrous) and level of acuity (with 2-point thresholds on the finger 15 times smaller than on the thigh). Whether these factors or the characteristics of the procedures and devices themselves account for the differences seen in the measures of discrimination and masking is unknown. 
The results of these studies suggest that, unless specific tests are made at different sites, a general conclusion regarding the relative efficacy of processing capabilities cannot be made. For example, although pattern discrimination appears to be superior on the thigh relative to the palm and finger for longer pattern durations, it is clearly inferior for shorter patterns. In this situation, the finger appears to be the most resistant to the influence of pattern duration, with performance depending solely on the temporal interval between pattern onsets. These results are not predictive of those obtained in the patternrecognition experiments. A completely different ordering of performance would be predicted for discrimination if it were based on pattern recognition.

The conclusion to be drawn from these measurements is perhaps not surprising: Using the results obtained from one site of stimulation, it is not possible to predict precisely the results that will be obtained from another site. Yet, the same general processes seem to be at work across sites. In Experiment 1, discrimination performance generally improved with increasing temporal separation between patterns, and SOA appeared to be a better descriptor of the function than did ISI. The overall level of discriminability, however, changed with site of stimulation, which in turn interacted with pattern duration. Similarly, and not surprisingly, in Experiment 2 all sites tested on the identification task did show some improvement with practice, but again the overall levels of performance vary from site to site and interact with pattern duration. As with the discrimination task, longer pattern duration produced better recognition, particularly on the thigh and palm. The results from Experiment 3 showed more backward than forward masking, although the difference between forward and backward masking is not as great on the thigh as it is on the finger, and the slopes of the two forward masking functions may differ.

There seems little in previous work that would have predicted the outcome of these measurements. Perhaps the most readily understood are the identification results (Experiment 2). The rank ordering of overall recognition scores, finger followed by palm and thigh, might be predicted based on measures of absolute sensitivity, spatial acuity, and density of innervation. As already noted, however, even here one might imagine obtaining a different ordering of results if the "10 easy patterns" were designed to be as highly recognizable by the palm and thigh as by the finger. By selecting patterns designed for a particular site, the variability associated with initial errors resulting from confusions with other patterns in the set is certainly minimized, if not eliminated. It is likely that the poor performance of the palm and thigh seen in Experiment 2 reflects just this problem. On the other hand, the nature of the discrimination task (Experiment 1) does not require correct absolute identification of either pattern in the pair in order to achieve high scores, only that the observer respond to any difference felt.

Underlying these conclusions is the notion that in the process of designing patterns, the concept of "site of stimulation" includes both the site itself and the stimulator array that will be used. The design of the array, that is, its size and shape, is somewhat constrained by the site. But with new types of arrays, one could increase the number of stimulators within a given area (although that might not necessarily improve pattern presentation; Daley \& Singer, 1975), or design a stimulator that wrapped around the limb, increasing the effective area of stimulation for a site. It is clearly not possible to define an "ideal" stimulator independent of the site where it will be used or independent of some measure of performance. That being the case, it becomes very difficult to attribute differences in performance to the site, to the stimulator, or to the types of patterns used. In effect, pattern identity is a "psycho-metric" measure. That is, the identity depends to some degree on the geometric properties of the "pattern," but also on its temporal parameters, temporal and spatial association with other presentations, and the interaction of the display device with the skin site in order to produce some unique constellation of activity that is tactually memorable.

There are some obvious difficulties in using the present results to select a site for a cutaneous communication system. First, as just noted, the type of display and the kinds of patterns that are to be used are likely to influence the results. These might well change the ordering of performance levels among sites as well as how pattern duration interacts with the variable of site. Thus, the ordering of sites for identification of the spatial patterns from best to poorest is finger, palm, thigh; but for discrimination of longer patterns it is thigh, finger, palm, with the thigh moving to third position if the patterns are brief. One conclusion is that if the communication system simply requires the subject to respond to some change in stimulation, that is, perform a discrimination, and the patterns are fairly long in duration, the thigh would be a good candidate. Such a system might be useful as an alerting device. If pattern recognition is required, however, the fingers would be the first choice. One could imagine a hybrid system using displays at the thigh and the finger with discrimination and identification tasks divided accordingly. Nevertheless, continued study across sites with comparable patterns, devices, and paradigms should be encouraged, for only in this way can additional commonalities and differences be disclosed.

\section{REFERENCES}

BACH-Y-RITA, P. (1970). Neurophysiological basis of a tactile vision-substitution system. IEEE Transactions on Man-Machine Systems, MMS-11, 108-109. 
BLiss, J. C. (Ed.) (1970). Tactile displays conference. IEEE Transactions on Man-Machine Systems, MMS-11 (Whole No. 1).

Brooks, P. L., \& Frost, B. J. (1983). Evaluation of a tactile vocoder for word recognition. Journal of the Acoustical Society of America, 74, 34-39.

Cholewiak, R. W. (1979). Spatial factors in the perceived intensity of vibrotactile patterns. Sensory Processes, 3, 141-156.

Cholewiak, R. W., \& Sherrick, C. E. (1981). A computercontrolled matrix system for presentation to the skin of complex spatiotemporal patterns. Behavior Research Methods \& Instrumentation, 13, 667-673.

CRalG, J. C. (1977). Vibrotactile pattern perception: Extraordinary observers. Science, 196, 450-452.

Craig, J. C. (1980). Modes of vibrotactile pattern perception. Journal of Experimental Psychology: Human Perception and Performance, 6, 151-166.

Craig, J. C. (1983). The role of onset in the perception of sequentially presented vibrotactile patterns. Perception \& Psychophysics, 34, 421-432.

Craig, J. C., \& Sherrick, C. E. (1982). Dynamic tactile displays. In W. Schiff \& E. Foulke (Eds.), Tactual perception: $A$ sourcebook (pp. 209-233). Cambridge, England: Cambridge University Press.

Daley, M. L., \& Sinaer, M. (1975). A spatial resolution measure of cutaneous vision. IEEE Transactions on Systems, Man, and Cybernetics, SMC-5, 124-125.

Enomlmann, S., \& Rosov, R. J. (1975). Tactual hearing experiment with deaf and hearing subjects. Journal of Exceptional Children, 41, 245-253.

Gault, R. H. (1927). "Hearing" through the sense organs of touch and vibration. Journal of the Franklin Institute, 204, 329-358.

Geldand, F. A. (1975). Sensory saltation: Metastability in the perceptual world. Hillsdale, NJ: Erlbaum.

Geldard, F. A. (1982). Saltation in somesthesis. Psychological Bulletin, 92, 136-175.

Geldard, F. A., \& Sherrick, C. E. (1972). The cutaneous "rabbit": A perceptual illusion. Science, 178, 178-179.
Green, B. G. (1982). The perception of distance and location for dual tactile pressures. Perception \& Psychophysics, 31, 315-323.

Loomis, J. M. (1980). Interaction of display mode and character size in vibrotactile letter recognition. Bulletin of the Psychonomic Society, 16, 385-387.

McFAdDEN, D. (1970). Three computational versions of proportion correct for use in forced-choice experiments. Perception \& Psychophysics, 8, 336-342.

Saunders, F. A. (1974). Electrocutaneous displays. In F. A. Geldard (Ed.), Cutaneous communication systems and devices (pp. 20-26). Austin, TX: Psychonomic Society.

Scadden, L. A. (1973). Tactile pattern recognition and body loci. Perception, 2, 333-336.

Sherrick, C. E., \& Rogers, R. (1966). Apparent haptic movement. Perception \& Psychophysics, 1, 175-180.

Sparks, D. W., Kuhl, P. K., Edmonds, A. E., \& Gray, G. P. (1978). Investigating the MESA (Multipoint Electrotactile Speech Aid): The transmission of segmental features of speech. Journal of the Acoustical Society of America, 63, 246-257.

SwETs, J. A. (Ed.) (1964). Signal detection and recognition by human observers. New York: Wiley.

VERRILLO, R. T. (1966). Vibrotactile thresholds for hairy skin. Journal of Experimental Psychology, 72, 47-50.

VERRILLO, R. T. (1974). Vibrotactile intensity scaling at several body sites. In F. A. Geldard (Ed.), Cutaneous communication systems and devices (pp. 9-14). Austin, TX: Psychonomic Society.

WEBER, E. H. (1978). The sense of touch. (De Tactu. H. E. Ross, Trans., \& Der Tastsinn, D. J. Murray, Trans.). New York: Academic Press. (Original work published 1826).

Weinstein, S. (1968). Intensive and extensive aspects of tactile sensitivity as a function of body part, sex, and laterality. In D. R. Kenshalo (Ed.), The skin senses (pp. 195-222). Springfield, IL: Thomas.

(Manuscript received January 17, 1984;

revision accepted for publication March 16, 1984.) 